\title{
ARTÍCULO
}

\section{PANDEMIA Y GOBIERNOS LOCALES EN EL INTERIOR DE LA PROVINCIA DE BUENOS AIRES: EL REIMPULSO INTERNACIONALIZADOR ${ }^{1}$}

\section{Resumen}

\author{
Julieta Nicolao* \\ Mariana Calvento** \\ Analuz Piersanti****
}

El objetivo del artículo es realizar una reflexión empíricamente informada en torno al impacto reciente de la pandemia por el COVID-19 sobre las estrategias de inserción internacional de los gobiernos locales. La hipótesis que orienta el análisis asume que se observa una incidencia positiva de la crisis sanitaria global sobre los intereses y motivaciones, así como en las herramientas disponibles para las administraciones locales de cara a su accionar internacional.

El trabajo, sustentado en un amplia revisión y análisis documental, centrado en los gobiernos municipales del interior de la Provincia de Buenos Aires en base a las experiencias de Tandil y Pergamino, revela que ambos se sirvieron de su trayectoria de internacionalización logrando no sólo sostener una agenda activa en este plano, sino también, incluyendo las relaciones internacionales como parte de la actuación local para enfrentar la crisis. En ese sentido, mientras Pergamino se recostó más en sus políticas unilaterales de comercio exterior, Tandil mantuvo una performance más integral, con énfasis en sus relaciones multilaterales, a través de la participación en redes de ciudades.

Palabras clave: relaciones internacionales, gobiernos locales, pandemia, Provincia de Buenos Aires

\footnotetext{
${ }^{1}$ Este artículo se ha elaborado en el marco de los siguientes proyectos de investigación: Relaciones internacionales y actores subnacionales: dinámicas, estrategias y políticas en el interior de la Provincia de Buenos Aires (Cod. 03/D316). Acreditado en el Programa de Incentivos a Docentes - Investigadores Secretaría de Políticas Universitarias - Ministerio de Educación (2019-2022); y Relaciones internacionales y gobiernos subnacionales. Estudio comparativo de municipios del interior de la República Argentina (Cod. PICT 2019-01165). Seleccionado y financiado en el marco de la Convocatoria 2019 Temas Abiertos, adjudicado según Res-2021-15-APN-DANPIDTYI\#ANPIDTYI (2021-2023).

* Lic. en Relaciones Internacionales y Dra. en Ciencia Política. Investigadora Adjunta de la Comisión de Investigaciones Científicas de la Provincia de Buenos Aires (CIC) con lugar de trabajo en el CEIPIL de la UNICEN, Tandil, Argentina. E-mail: nicolao_j@yahoo.com.ar. ORCID: 0000-0002-8083-5301.

** Lic. en Relaciones Internacionales y Dra. en Ciencia Política. Investigadora Adjunta del Consejo Nacional de Investigaciones Científicas y Técnicas (CONICET) con lugar de trabajo en el CEIPIL de la UNICEN, Tandil, Argentina. Profesora Adjunta de la Licenciatura en Relaciones Internacionales (FCHUNICEN). E-mail: marianacalvento@yahoo.com.ar. ORCID: 0000-0002-0257-3270.

*** Lic. en Relaciones Internacionales. Auxiliar en proyectos de investigación acreditados del CEIPILUNICEN-CIC. E-mail: anitapier.08@gmail.com. ORCID: 0000-0002-5697-7393.
} 


\title{
PANDEMIC AND LOCAL GOVERNMENTS IN THE INTERIOR REGION OF THE PROVINCE OF BUENOS AIRES: THE INTERNATIONALIZING REIMPULSE
}

\begin{abstract}
The objective of the article is to carry out an empirically informed reflection on the recent impact of the COVID-19 pandemic on the international insertion strategies of local governments. The hypothesis that guides the analysis assumes that a positive impact of the global health crisis is observed on the interests and motivations, as well as on the tools available to local administrations for their international actions.

The work, based on an extensive review and documentary analysis, focused on the municipal governments of the interior of the Province of Buenos Aires based on the experiences of the municipalities of Tandil and Pergamino, reveals that both used their internationalization trajectory, achieving no not only to support an active agenda in this area, but also, including international relations as part of local action to face the crisis. In this sense, while Pergamino leaned more on its unilateral foreign trade policies, Tandil maintained a more comprehensive performance, with emphasis on its multilateral relations, through participation in city networks.
\end{abstract}

Keywords: international relations, local governments, pandemic, province of Buenos Aires

TRABAJO RECIBIDO: 02/09/2021 - TRABAJO ACEPTADO: 20/11/2021 


\section{Introducción}

El objetivo de este trabajo es realizar una reflexión empíricamente informada en torno al impacto reciente de la pandemia por el COVID-19 sobre las estrategias de proyección internacional de los gobiernos municipales del interior de la Provincia de Buenos Aires. La hipótesis que orienta el análisis asume que, lejos de un efecto paralizador, se observa una incidencia positiva de la crisis sanitaria global sobre los intereses y motivaciones, las herramientas y los recursos disponibles para las administraciones locales en su accionar internacional en sentido amplio. Este reimpulso internacionalizador se asocia a la propia necesidad de superar la crisis sanitaria (y socioeconómica) que ha conducido a algunos gobiernos subnacionales a involucrarse en plataformas, redes globales, alianzas internacionales entre municipios y/o acuerdos bilaterales para generar esquemas colaborativos o de cooperación descentralizada en la lucha contra el covid-19, facilitados, entre otros condicionantes, por la arrolladora digitalización de las comunicaciones y la necesidad de buscar recursos externos para reemplazar aquellos ingresos tributarios locales (o producto de la transferencia del Estado central) que se vieron mermados por la crisis.

Para mostrar evidencia empírica sobre la tendencia expuesta, el estudio abarca temporalmente el registro de experiencias y actividades generadas en dos municipios de tamaño intermedio ${ }^{1}$ del interior bonaerense entre marzo de 2020 hasta la actualidad (el Municipio de Tandil y el Municipio de Pergamino). La selección de los mismos responde a que diversos estudios confirman una participación significativa de los gobiernos locales de dicha provincia en el escenario internacional (Castro y Saslavsky, 2009; Pontis, 2012; Calvento, 2012; 2020; entre otros); y en el área del interior bonaerense los casos escogidos han sido identificados con un perfil continuo y alto en su performance internacional en el período pre-pandémico, aspectos que validan la importancia de analizar sus experiencias.

El artículo se sustenta en el registro, relevamiento y análisis de estrategias de internacionalización multilaterales, bilaterales y unilaterales. Dada la naturaleza reciente del proceso analizado y de la ausencia de producción científica sobre la temática en general, y de este espacio geográfico en particular, para la reconstrucción de cada experiencia se apeló a la consulta y análisis de un amplio corpus documental constituido por ordenanzas, informes técnicos y estadísticas producidas por los gobiernos locales; prensa oficial municipal y sectorial; planes estratégicos municipales; informes de gestión del gobierno de la Provincia de Buenos Aires, medios de comunicación de las propias redes internacionales de ciudades, entre otros. Para el diseño del marco teóricoconceptual se consultó bibliografía especializada en la materia, informes de organismos y agencias internacionales, y la relatoría de los principales foros y conferencias globales sobre la internacionalización de las ciudades o los gobiernos locales en tiempos de pandemia.

El artículo se estructura en tres segmentos: el primero se ocupa de desarrollar los principales lineamientos teórico-conceptuales que definen la investigación sobre las relaciones internacionales de los municipios (sus dimensiones, estrategias, tipologías, etc.); el segundo sintetiza alguna de las dimensiones centrales del actual debate en torno

${ }^{1}$ Contienen una población de 50.000 a 1.000 .000 habitantes (Vapñarsky y Gorojovsky, 1990; Manzano y Velázquez, 2015). 
a los retos que ha presentado la pandemia para las ciudades y los gobiernos no centrales en materia de cooperación e inserción internacional; el tercero y último presenta las experiencias de los dos distritos seleccionados.

\section{Aproximaciones teórico-conceptuales sobre la internacionalización de los gobiernos locales}

En las últimas décadas, los gobiernos municipales han debido afrontar nuevos desafíos, producto de los procesos socioeconómicos y políticos contemporáneos ampliamente desarrollados en estudios sobre el tema (Borja y Castells, 1997; Batista, Jakobsen, y Evangelista, 2008; Álvarez, Luna Pont y Oddone, 2019; entre otros), que han actuado sobre ellos, impactando e influyendo sobre sus funciones, sus recursos y sus identidades, lo que se ha visto potenciado por el contexto de pandemia. Estos procesos han tornado más complejo su rol político-administrativo, colocándolos en el centro de los debates y las demandas de la sociedad, así como acentuando su relevancia en la gestión e implementación de estrategias de desarrollo local e internacional. En ese sentido, pese a no ser considerados como sujetos de derecho internacional, desarrollan alternativas de participación en el escenario mundial utilizando herramientas de cooperación internacional, estableciendo acuerdos económicos con representantes extranjeros, integrando redes globales de ciudades, organizando misiones comerciales, promoviendo su potencial exportador, entre otros instrumentos.

De esta manera, presentan diversas modalidades de participación internacional con el principal objetivo de establecer vínculos que le permitan acceder a un conjunto de recursos materiales (económico-comerciales, financieros, tecnológicos) e inmateriales (contactos, experiencias, buenas prácticas), que brinda el escenario internacional con la finalidad de que los mismos colaboren en la estrategia de desarrollo local definida. Los programas y proyectos que se generan y materializan en el marco de este accionar internacional colaboran en áreas tan variadas como la economía, la salud, el medio ambiente, así como en la modernización administrativa local, entre tantos otros.

Desde la disciplina de las Relaciones Internacionales se han desarrollado numerosos conceptos para denominar la performance internacional de los gobiernos subnacionales, de los cuales se destaca la relevancia y alcance extendido que ha tenido el término paradiplomacia (Duchacek, 1986; Michelmann y Soldatos, 1990) estableciéndose como el de mayor uso y aceptación. En trabajos previos (Calvento, 2012, 2016b, 2020) se han desarrollado consideraciones sobre la paradiplomacia proponiendo un concepto alternativo como el de Política Internacional Subnacional (Maira, 2010) definida específicamente como la herramienta de los gobiernos locales que se ocupa de impulsar su inserción internacional, permitiéndole aprovechar, articuladamente, las oportunidades del contexto exterior con las necesidades del territorio, dentro de los marcos que define la política exterior del Estado nacional. Así se la entiende como una política pública local, que materializa y aplica la estrategia de un gobierno de este nivel en el campo de las Relaciones Internacionales.

Partiendo de esta base, se pueden distinguir diferentes estrategias dentro de la política internacional subnacional que los gobiernos municipales han implementado para conectarse al plano global. Estas pueden sistematizarse a través de tres 
modalidades: unilateral, bilateral y multilateral. Las mismas sintetizan las numerosas experiencias y herramientas reseñadas por diferentes autores (Borja y Castells, 1997; Romero, 2004; Vigevani, 2006; Salomón, 2007; Batista, Jakobsen, y Evangelista, 2008; Colacrai, 2013; Marcovitch y Dallari, 2014; Carignani Sardoy, 2017; Prado Figueredo, 2018; Álvarez, Luna Pont y Oddone, 2019; Schiavon, 2020; entre otros) abarcando estrategias de presencia directa e indirecta, principalmente de carácter formal, que desarrollan en el ámbito regional y global.

En primer lugar, están las estrategias de carácter unilateral que establecen como objetivo la promoción y posicionamiento en el exterior de los actores subnacionales. Éstas implican un mayor compromiso por parte de los gobiernos municipales ya que, a diferencia de las bilaterales y multilaterales, son herramientas que dependen exclusivamente de la decisión y trabajo unilateral de estos actores. Visualizan la tendencia creciente del involucramiento de los gobiernos locales en el desarrollo productivo e integral orientado hacia afuera, e incluyen el desarrollo de instrumentos locales que impulsan el comercio exterior, la implementación de políticas de posicionamiento estratégico que promueven el establecimiento de una imagen territorial como la Marca Ciudad, entre otras.

En segundo lugar, las herramientas bilaterales se concretan a través de acuerdos entre ciudades o regiones y entre ciudades e instituciones/organismos internacionales. Las estrategias más utilizadas dentro de este tipo son los hermanamientos de ciudades, aunque también pueden registrarse otras modalidades como actas y convenios de cooperación internacional bilateral, acuerdos transfronterizos, así como las visitas de las autoridades locales al exterior y la recepción de autoridades extranjeras. Las temáticas que las definen abarcan diferentes aristas como la formación de recursos humanos, la modernización administrativa, la mejora de infraestructura pública, la innovación de los sectores productivos a través del fomento a las PyMEs, el impulso de la protección del medio ambiente y la promoción de la cultura, el turnismo, etc. La modalidad de los acuerdos permite que los gobiernos subnacionales logren el acceso a la asistencia técnica, financiera, entre otras.

Por último, las multilaterales se presentan, principalmente, bajo el formato de redes de ciudades. Una característica transversal que las distingue es que se establecen a partir de relaciones horizontales, ya que se realizan entre instituciones similares sin jerarquía, y cuentan con un mayor margen de flexibilidad y dinamismo que las estrategias de carácter bilateral (Zapata Garesché, 2007). En las relaciones multilaterales se distinguen las regionales de las globales, tanto por sus integrantes como por los objetivos que se proponen. De esta manera, dentro de las regionales se encuentran, por ejemplo, la Red de Mercociudades, la Federación Latinoamericana de Ciudades, Municipios y Asociaciones de Gobiernos Locales (FLACMA); en tanto que, en las globales, puede citarse a la organización Ciudades y Gobiernos Locales Unidos (CGLU), entre otras. También se encuentran aquellas redes temáticas como la Asociación Internacional de Ciudades Educadoras (AICE), Gobiernos Locales por la Sostenibilidad (ICLE por sus siglas en inglés). Incluso se desarrollan otros espacios de vinculación multilateral como la Coordinación de Autoridades Locales de América Latina (CORDIAL), creada en 2015 como una plataforma que reúne a las principales 
redes de ciudades ${ }^{2}$ y Gobiernos locales de Iberoamérica; y otras que se detallan en segmentos subsiguientes como Cities for Global Health.

Así, a través de estas estrategias unilaterales, bilaterales y multilaterales, los gobiernos locales acceden a la formación de recursos humanos de quienes integran las estructuras técnicas y administrativas en temáticas de interés; al financiamiento para proyectos en distintas áreas; al conocimiento de otras experiencias y prácticas para la resolución de problemas comunes; a la ejecución de proyectos orientados al territorio en acuerdo con otras instituciones y actores extra-territoriales; a ejercer cierta influencia política, etc. (Batista, Jakobsen y Evangelista, 2008; Maira, 2010; Cravacuore, 2015). Por estos, entre otros motivos, las relaciones internacionales de los gobiernos locales adquieren cada vez mayor importancia y visibilidad, potenciada en el contexto actual.

\section{La pandemia y el reimpulso internacionalizador}

Desde la declaración de la Organización Mundial de la Salud en la cual se definió al COVID-19 como pandemia, la política internacional ha afrontado dos tendencias simultáneas: una tendencia a la re-centralización protagonizada por los gobiernos nacionales ${ }^{3}$, que se han ocupado de atender el control soberano del territorio y la población, el cierre de fronteras internacionales, la restricción de la circulación interna, aislamiento social y control ciudadano con una lógica operatoria "fronteras adentro" y apelando en gran medida a discursos nacionalistas; y otras fuerzas internacionalistas asentadas en un espíritu de solidaridad y cooperación global, que han buscado esquemas colaborativos internacionales que permitan contener el avance y reducir el impacto negativo del virus en todas las regiones del mundo, tendencia más anclada en el accionar horizontal de ciudades y gobiernos subnacionales (AL-LAs y OCD, 2020; Juste, 2020).

Las ciudades, en rigor, desde el surgimiento de la crisis sanitaria global que tuvo su epicentro en Wuhan (China), han visto resignificada su importancia y gravitación a escala internacional. El impacto del COVID-19 en las grandes urbes con mayor densidad poblacional y de interconectividad (donde los niveles de contagio y las tasas de letalidad del virus adquirieron valores alarmantes) interpeló directamente a los gobiernos de las ciudades, en tanto instancias estatales que actúan en la "primera línea de respuesta" sobre la población a su cargo (Organización de las Naciones Unidas, 2020).

En la coyuntura pandémica se han visto entonces modificados en gran medida los modelos de gobernanza local, al igual que la dinámica de las relaciones multinivel (con órdenes o niveles superiores de gobierno) y en algunos casos también, las vinculaciones y estrategias de cooperación internacional que conducen las administraciones locales.

\footnotetext{
${ }^{2}$ Participan la Alianza Euro-Latinoamericana de Cooperación entre Ciudades (AL-LAs), la Federación Latinoamericana de Ciudades Municipios y Asociaciones de Gobiernos Locales (FLACMA), la Red de Mercociudades y la Unión de Ciudades Capitales Iberoamericanas (UCCI).

${ }^{3}$ Vale aclarar que, el contexto de pandemia, en este caso, potencia un proceso que había comenzado a desarrollarse con anterioridad. Ver: Cravacuore, D. (2017). La recentralización municipal en la Argentina. Estado Abierto. Revista sobre el Estado, la administración y las políticas públicas, 2 (1), 167-190.
} 
Este reimpulso internacionalizador ha tenido diferentes variables: por un lado, la necesidad de obtener legitimidad política interna para las autoridades no centrales, sobre todo para aquellas cuyos gobiernos nacionales adoptaron una postura contrapuesta al propio abordaje de la crisis sanitaria. A nivel latinoamericano emerge el caso emblemático de Brasil, con un gobierno federal que negó la magnitud del impacto de la pandemia, dejando a muchos gobiernos subnacionales brasileños expuestos a la necesidad de empoderarse internacionalmente para imprimirle legitimidad a la toma de decisiones a escala local (ONU, 2020).

En segundo término, la amplia digitalización de los procesos sociales y de las comunicaciones, ha resultado un elemento facilitador para la participación internacional, incluso para gobiernos locales que se involucran por primera vez en experiencias multilaterales:

(...) la crisis provocada por el virus ha producido un efecto innovador en el accionar internacional subnacional, originando acciones de cooperación a través de la virtualidad dada la imposibilidad de reuniones presenciales. Este último hecho, a su vez, impulsó el accionar internacional de unidades subnacionales de menor desarrollo a las que se les dificulta ejecutar una agenda internacional por los costos que representan las modalidades tradicionales de cooperación que incluyen una dinámica de intercambios físicos que no pueden afrontar (Juste, 2020: p. 4).

Este punto también abre muchos interrogantes respecto a lo que sucederá en un futuro retorno a lo que se ha denominado sociedad postpandémica o "nueva normalidad", y si estas dinámicas virtuales con impacto inclusivo perdurarán en el tiempo o resultarán velozmente reemplazadas por las prácticas tradicionales de la "presencialidad".

Otro elemento traccionador ha sido la búsqueda unilateral o multilateral de recursos (económicos) para poder ejercer las abultadas responsabilidades que han asumido los gobiernos subnacionales ante la crisis en materia de prevención y respuesta, contención económica y social, entre otras. En relación a ello, se ha observado que tanto la crisis sanitaria como las medidas de restricción circulatoria y de aislamiento social provocaron el cierre de comercios, la pérdida de fuentes de trabajo y otras consecuencias económicas desfavorables que afectaron los ingresos tributarios que perciben los municipios, colocando sus arcas financieras en una situación crítica. Más aún, "Los lugares donde la base económica está menos diversificada están sufriendo las repercusiones de modo especial. Por ejemplo, las ciudades cuyos ingresos dependen fundamentalmente del turismo" (ONU, 2020, p. 2). Ante este panorama, la cooperación internacional descentralizada, la posibilidad de acceder a donaciones $\mathrm{o}$ fondos especiales de ayuda, empuja con mayor determinación a las unidades subnacionales al escenario internacional.

En cuarto término, ha resultado favorecedor la complejidad de la internacionalización territorial que se produjo en algunos espacios subnacionales donde un entramado cada vez más amplio de actores como universidades, centros de investigación y desarrollo tecnológico, agencias de desarrollo, empresas y fundaciones privadas promovieron diálogos, intercambios y cooperación internacional (entre cuyos canales circularon recursos económicos, financieros, humanos, de conocimiento, 
equipamiento sanitario, etc.) (Hidalgo, 2021). Esto ha conducido, cuando no obligado, a los gobiernos locales a acompañar, reconocer, formalizar, los esfuerzos conducidos por actores locales extra-municipales, involucrándose de este modo en dichos procesos.

Ahora bien, en lo que concierne a las modalidades en que los gobiernos municipales que participaron de esta tendencia han materializado sus relaciones internacionales desde el inicio de la pandemia, se ha reconocido que, por un lado, las estrategias no se han modificado, sino que se sostienen las ya existentes (con readaptaciones o renovaciones en función de esta nueva coyuntura). En este punto Nahuel Oddone adelanta que "Las ciudades hermanas y las redes de autoridades locales parecen ser las ganadoras dentro de las actividades paradiplomáticas desde el comienzo de la pandemia" (2021, p.1), sin embargo, como se observará en el último apartado de este artículo, las experiencias analizadas distan entre sí y ofrecen un panorama heterogéneo.

En relación a ello, algunos estudios reconocen una intensificación de los intercambios bilaterales entre entidades subnacionales con previos lazos establecidos (hermanamientos, convenios de cooperación) o entre gobiernos locales y organizaciones internacionales (Hidalgo, 2021). Pero también se ha visibilizado un engrosamiento del trabajo en red multilateral para el fortalecimiento de capacidades hacia la generación de respuesta a la crisis sanitaria. En rigor, diversas redes de ciudades preexistentes adaptaron sus estructuras, dinámicas de funcionamiento y agendas para generar rápidamente espacios de intercambio de información, conocimiento y buenas prácticas, protocolos o planes de acción específicos en la lucha contra el COVID-19. Y también se han gestado alianzas entre las propias redes internacionales de ciudades.

Esto último se reconoce como un aspecto positivo, en tanto los diagnósticos de la situación pre-pandémica daban cuenta de una importante confluencia de redes de ciudades con demandas de participación masiva en espacios internacionales, que solapaban sus actividades en tiempo y espacio, y que habían generado cierta saturación a su público objetivo, sin tampoco evidenciar resultados claros sobre los beneficios de participación en las mismas. En tal sentido, se viene observando que las redes han aunado esfuerzos y fusionado estrategias, absorbiendo demandas de eficiencia mayores. Al menos esto ha comenzado a formar parte de los debates que se suscitan en estos espacios (AL-LAs y OCD, 2020).

Algunos ejemplos: CGLU y la Asociación Mundial de las Grandes Metrópolis (comúnmente denominado Metrópolis) organizaron con velocidad sesiones de formación en vivo y pusieron en marcha junto a la Alianza Euro-Latinoamericana para la Cooperación entre Ciudades (AL-LAS), la plataforma Cities for Global Health. Ésta constituye un espacio de intercambio de información entre gobiernos locales en las que están representados actualmente 34 países y 105 ciudades del mundo, siendo libre el acceso a la información que es compartida (más de 680 iniciativas ${ }^{4}$ de prevención o preparación, respuesta y recuperación ante el COVID-19).

Otra de las redes internacionales que emergieron durante la pandemia fue Cities for a Resilient Recovery (Ciudades para una recuperación resiliente), desarrollada en el marco de la más amplia Red de Ciudades Resilientes (Resilient Cities Network). La particularidad de su membresía para el caso que nos ocupa es que, en la actualidad,

\footnotetext{
${ }^{4}$ Número actualizado a septiembre de 2021. https://www.citiesforglobalhealth.org/
} 
participan sólo grandes ciudades y metrópolis del mundo, incluyendo solamente dos ciudades argentinas: la Ciudad Autónoma de Buenos Aires y la ciudad de Santa Fe. También desde otros espacios como ICLEI, Commonwealth Local Government Forum, Mayors Migration Council, C40, Eurocities, entre otros, se han facilitado actividades de formación, de trabajo en red, compartiendo orientaciones e instrumentos múltiples.

Ahora bien, uno de los ámbitos centrales para visualizar el desarrollo de estrategias de tipo multilateral para gobiernos locales bonaerenses en el contexto pandémico, es el ámbito de la integración regional mercosureña, y en el marco de la misma, Mercociudades. Desde este espacio se puso en funcionamiento la estrategia "Mercociudades responde al COVID-19", cuyo objetivo es servir de orientación para aquellas ciudades que necesiten encontrar sus propias soluciones en la lucha contra la crisis sanitaria. Se asienta en tres pilares fundamentales: la generación de herramientas de comunicación específicas (página Web, redes sociales, etc.); la cooperación solidaria entre ciudades miembro y las diversas instancias de coordinación de Mercociudades; y la articulación y alianza con terceros para compartir conocimientos, co-crear herramientas, sensibilizar e incidir.

En la página Web creada a tal efecto ${ }^{5}$ se dispusieron cientos de iniciativas de utilidad para los gobiernos locales, entre las que se incluyen experiencias locales en el combate del virus, seminarios Web, orientaciones para el teletrabajo, circuitos de solidaridad $^{6}$, convocatorias para el financiamiento de proyectos destinados a paliar las consecuencias del coronavirus, material actualizado sobre la pandemia y cómo afrontarla, y un espacio específico para solicitar colaboración en la formulación de proyectos.

En el marco expuesto, para muchos gobiernos locales de la Provincia de Buenos Aires la participación en estos espacios multilaterales ha sido un canal privilegiado para acceder a experiencias, conocimientos y prácticas desarrolladas en distintas zonas urbanas de todo el mundo, o de compartir las propias iniciativas.

\section{Experiencias bonaerenses}

\section{Pergamino}

Pergamino es un municipio de tamaño intermedio ubicado en el norte de la Provincia de Buenos Aires, que se encuentra a $222 \mathrm{~km}$ de la Ciudad Autónoma de Buenos Aires (CABA) y a $30 \mathrm{~km}$ del límite con la Provincia de Santa Fe. Pertenece a la región interior de la provincia y según datos derivados del último Censo Nacional de Población (2010), cuenta con 104.590 habitantes, asentados en un $87 \%$ en su ciudad cabecera homónima y el resto distribuidos en los doce pueblos de campaña que componen el Partido y su zona rural (INDEC, 2010). Se ubica en la llanura pampeana, en la porción correspondiente a la pampa ondulada, en la cuenca del Arroyo Pergamino, y su economía está en gran medida ligada a la actividad agropecuaria (fundamentalmente agrícola) y un importante sector industrial (confeccionista,

\footnotetext{
${ }_{6}^{5}$ https://mercociudades.org/covid-19/

6 Los circuitos solidarios consistieron en iniciativas compartidas por municipios, universidades, organismos internacionales, alianzas de actores territoriales que han propuesto líneas de acción que pueden ser emuladas en otras latitudes. A septiembre de 2021 existían 60 iniciativas.
} 
maderero, metalúrgico, plástico y agroalimentario) (Municipalidad de Pergamino, 2011, 2018). Figuran entre los actores clave del territorio, instituciones de educación superior y científico-tecnológicas como la sede de la Universidad Nacional del Noroeste de la Provincia de Buenos Aires (UNNOBA) y el INTA Estación Experimental Pergamino; instituciones reconocidas del campo de la salud pública; el Parque Industrial y un amplio entramado de organizaciones de la sociedad civil (deportivas, educativas, sociales y culturales) que enriquecen y densifican la vida comunitaria del distrito (CFI, 2017). También es un partido que se distingue por su ubicación geográfica privilegiada, a la vera de las rutas Nacionales 8 y 188 y la Ruta Provincial 178, lo cual le habilita una óptima conectividad con el resto de la provincia bonaerense y las provincias vecinas, a escasa distancia de la Hidrovía Río Paraná, y con una ágil accesibilidad a dos de las ciudades más importantes de la Argentina: Rosario y CABA (Municipalidad de Pergamino, 2011).

Este municipio cuenta con una trayectoria de internacionalización definida por una diversidad de estrategias y herramientas que, en la mayoría de los casos, encuentran sus antecedentes en el decenio de 1990. Según la categorización establecida por Calvento (2012), en función de la aplicación del índice de participación internacional ${ }^{7}$, Pergamino se ubicaba para 2012 dentro del conjunto de municipios con perfil de internacionalización "alta", que contempla aquellas experiencias donde confluye el desarrollo de estrategias de gestión internacional de tres tipos (unilaterales, bilaterales y multilaterales).

En relación a las bilaterales, Pergamino ha incursionado desde la década del 90' en la firma de acuerdos con ciudades hermanas y, en la actualidad, cuenta con siete hermanamientos suscriptos con distintas contrapartes de la región latinoamericana y una china. El propósito central que ha motivado estas estrategias ha sido la promoción del desarrollo económico local; el impulso de proyectos con impacto territorial en ámbitos variados (seguridad ciudadana, seguridad vial, desarrollo sostenible, servicios públicos, promoción socio-cultural, inclusión, género, educación, etc.); y el intercambio de experiencias y recursos técnicos especializados sobre estos temas que permitan la propia capacitación y también realizar contribuciones a las contrapartes en función de la experiencia local adquirida (CFI, 2017).

En el ámbito multilateral y en lo que concierne específicamente a su participación en redes globales o regionales de ciudades, forma parte desde hace dos décadas (2001) de la Red Mercociudades. Pero no es ésta la única red de ciudades en la que participa, también integra la Asociación Internacional de Ciudades Educadoras (AICE), el Observatorio Internacional de Democracia Participativa, la Unión Iberoamericana de Municipalistas (IUM) y fue muy activo en su participación del Programa URB-AL (Programa de cooperación descentralizada entre autoridades subnacionales europeas y latinoamericanas de la Comisión Europea) que estuvo vigente hasta 2013 (Calvento 2012; CFI, 2017; Municipio de Pergamino, 2018). A partir de su participación en estas plataformas, pudo concretar diversos proyectos de impacto territorial.

\footnotetext{
${ }^{7}$ El Índice de Participación Internacional retoma las dimensiones unilateral, bilateral y multilateral de la política internacional subnacional e implica la construcción de una tipología que refleja categorías incrementales establecidas en relación a la internacionalización de los municipios intermedios a saber: de internacionalización baja, intermedia y alta. Esta última caracterizada por la existencia de estrategias de participación internacional de todas las dimensiones (Calvento, 2020).
} 
A nivel de estrategias unilaterales, en materia de política local de comercio exterior, Pergamino cuenta con un régimen de promoción de exportaciones propio, materia regulada vía ordenanzas $\mathrm{N}^{\circ} 2829 / 91$ y 5934/04: la primera establece beneficios de desgravaciones a empresas exportadoras locales que se radiquen en Pergamino, y la segunda otorga beneficios para la asistencia a eventos internacionales, misiones comerciales, rondas de negocios, etc. (Calvento 2012). En este campo, cuya trayectoria también se remonta a los años 90', el gobierno local ha venido trabajando en la orientación y acompañamiento al empresariado local, otorgándoles herramientas diversas para la búsqueda y aprovechamiento de las diferentes oportunidades comerciales a escala global. Al 2016, el distrito informaba vinculaciones comerciales bilaterales con 73 países, encabezando Brasil como principal comprador de sus productos con una distancia significativa de los siguientes: Uruguay, Italia, Países Bajos, Turquía, Estados Unidos, Pakistán, entre otros (Municipio de Pergamino, 2018).

Además de la dimensión de comercio exterior, cuenta con destacada experiencia en la organización de foros, congresos o eventos internacionales; una intensa actividad protocolar y de recepción de delegaciones extranjeras y visitas internacionales en su territorio, un elevado flujo e intercambio de estudiantes, entre otros (CFI, 2017).

Finalmente, para cerrar este recorrido, vale destacar que en el marco del Programa Bonaerense Municipios Internacionales ${ }^{8}$, Pergamino funcionó, en relación a su trayectoria de internacionalización, como un actor central en el proceso de transferencia de capacidades y difusión de mejores prácticas para el resto de los municipios participantes (CFI, 2017, p. 9). En rigor, dentro del diagnóstico de capacidades locales para la internacionalización, que significó una dimensión clave del citado programa, el municipio fue categorizado, según su nivel de apropiación y desarrollo de la acción internacional, en el Grupo A (integrado por municipios con mayor conocimiento y desarrollo de acciones internacionales). Por todo lo expuesto hasta aquí, puede advertirse que el distrito ingresó a la coyuntura pandémica con un plafón robusto de vinculaciones y estrategias internacionales.

\section{La internacionalización de Pergamino en tiempos pandémicos}

El gobierno municipal de Pergamino se encuentra bajo la gestión (2019-2023) de la misma fuerza política de la administración precedente (2015-2019), con un intendente reelecto perteneciente a la coalición Juntos por el Cambio, el abogado Javier Martínez. Esta continuidad no representa un dato menor al momento de la evaluación de la política de internacionalización en el período bajo estudio.

Desde el punto de vista institucional, la etapa analizada encuentra al gobierno local con dos departamentos con rango de dirección desde los cuales se gestionan las

\footnotetext{
8 Impulsado por la Dirección de Cooperación Internacional de la Provincia de Buenos Aires de la Secretaría General del gobierno bonaerense cuyo propósito fue contribuir al fortalecimiento de las capacidades locales para la inserción internacional de los municipios de la provincia. Para su implementación se convocaron originalmente a todos los municipios de la II sección electoral (incluyendo a Pergamino), luego ampliada a algunos distritos de la IV sección. Este Programa marca una continuidad con el Programa de Internacionalización de Municipios (PIM) lanzando a principios del año 2015 por la Subsecretaría de Relaciones Económicas Internacionales dependiente del Ministerio de la Producción, Ciencia y Tecnología de la Provincia de Buenos Aires (SREI, 2015).
} 
relaciones internacionales del Municipio, ambas dependientes de la Secretaría de Producción. Por un lado, la Dirección de Cooperación Internacional y Turismo que funciona como un área trasversal del resto de las áreas de gobierno desde las cuales se impulsa el establecimiento y desarrollo de las relaciones interinstitucionales con el exterior. Es decir que, este departamento tiene bajo su injerencia, "el desarrollo de acciones de vinculación internacional conforme a los principales lineamientos políticos locales y oficiando de facilitadora entre las diferentes dependencias municipales y el exterior"9. Por otro, la Dirección de Comercio Exterior (en adelante, DCE), que se incorporó en los 90' a la estructura municipal, y que centraliza el diseño e implementación de la política local de comercio exterior nutrida de un catálogo de herramientas que se utilizan en la articulación del municipio con el sector productivo y empresarial local: búsqueda de oportunidades comerciales (informes de mercado), perfiles de mercado; apoyo en la asistencia a ferias y misiones comerciales en distintos países y en el impulso de misiones comerciales inversas; agendas de negocios en viajes individuales; licitaciones internacionales; listado de importadores; producción de estadísticas de importación y exportación local propia, generación de base de datos de importadores y exportadores locales; asesoramiento y capacitación de empresarios, entre otros ${ }^{10}$.

El nivel de profesionalización de las áreas citadas puede vislumbrarse en el perfil profesional de las titulares de las mismas, formados en disciplinas directamente ligadas a la gestión internacional ${ }^{11}$.

Durante la crisis sanitaria, la agenda municipal en materia de internacionalización tuvo mayor activismo en la segunda de las áreas descriptas (DCE), lo cual se relaciona con un fuerte peso de estrategias unilaterales en este ámbito. Aprovechando la oleada de virtualización de las comunicaciones e instancias de diálogo y participación, la Dirección readaptó el grueso de sus herramientas habituales a la modalidad on line, desplegando una agenda con diversas actividades.

Una de las más significativas ha sido el desarrollo permanente de instancias de capacitación a cargo de especialistas, que fueron informando al público objetivo de la Dirección en temas relacionados con operatoria, logística, actualización pertinente, impacto del contexto mundial pandémico en el comercio exterior, y especialmente, oportunidades desconocidas en el mercado externo como alternativas para enfrentar la crisis.

\footnotetext{
${ }^{9}$ www.pergamino.gob.ar

10 www.pergamino.gob.ar

${ }^{11}$ La actual directora de Cooperación Internacional y Turismo es Lic. en Relaciones Internacionales, tiene un Máster en Comunicación y Periodismo, y una diplomatura en Gestión Municipal. La directora de Comercio Exterior es Analista en Computación Administrativa, Técnica Superior en Estadísticas de Empresas, y Técnica Superior en Comercio Exterior.
} 
Tabla 1: Listado de actividades de capacitación/actualización generadas desde la DCE

\begin{tabular}{|c|c|}
\hline Tema & Año \\
\hline El Comercio Exterior para recuperar la actividad post COVID. & 2020 \\
\hline Sin logística no hay comercio exterior. Sistemas logísticos integrados. & 2020 \\
\hline $\begin{array}{l}\text { Sin logística no hay comercio exterior. La estrecha vinculación entre Comex } \\
\text { y logística }\end{array}$ & 2020 \\
\hline Exportaciones sustentables: descubriendo República Dominicana & 2020 \\
\hline Retenciones a las exportaciones & 2020 \\
\hline Exportaciones sustentables: descubriendo Ruanda & 2020 \\
\hline Operatoria Cambiaria y novedades legales & 2020 \\
\hline Comercio Exterior Argentino & 2020 \\
\hline Panamá: la puerta del mercado centroamericano. Una herramienta de acceso. & 2020 \\
\hline Herramienta de acceso al mercado ruso y espacio post-soviético. & 2020 \\
\hline Licencias no automáticas de importación & 2020 \\
\hline Reintegros de exportación & 2021 \\
\hline El desafío de crecer y minimizar el riesgo de impago en exportaciones & 2021 \\
\hline Operatoria y novedades legales Parte I & 2021 \\
\hline Operatoria y novedades legales Parte II & 2021 \\
\hline Integración Regional y el MERCOSUR & 2021 \\
\hline
\end{tabular}

Fuente: elaboración propia en base a: https://comex.pergamino.gob.ar/ 
También se aprovechó esta dinámica virtual para la participación en plataformas o espacios internacionales de interés. Por ejemplo, Pergamino estuvo presente, a través de su intendente municipal, del $5^{\circ}$ Foro Mundial de Ciudades y Plataformas Logísticas, que tuvo sede en Paraguay en diciembre de 2020, pero se desarrolló de modo virtual. Organizado por la Organización Mundial de Ciudades y Plataformas Logísticas (OMCPL), contó con la participación de más de 15.000 profesionales logísticos de más de 60 países, y más de 70 conferencistas internacionales. El jefe comunal disertó en torno a "los municipios logísticos motores de la economía regional"12.

Del mismo modo, los intercambios bilaterales físicos o recepción de delegaciones extranjeras con fines de promoción del comercio exterior se reconvirtieron a la nueva virtualidad: la DCE participó de la Jornada de Trabajo denominada "Promoción del Intercambio Comercial" entre la Provincia de Buenos Aires y Bulgaria, organizado por el Departamento de Comercio de la UIPBA - Unión Industrial de la Provincia de Buenos Aires-, en la cual estuvieron presentes el Embajador de Bulgaria y el Presidente de la Cámara de Comercio de ese país, y diversas autoridades del gobierno provincial.

Otro de las líneas de gestión clave que se impulsaron y concretaron en pandemia fue el lanzamiento, a mediados de noviembre de 2020 de la página Web oficial de la Dirección de Comercio Exterior donde se condensa toda la información referida a la oferta exportable de Pergamino, las estadísticas producidas a nivel local en materia de exportación / importación, la información de mercados, etc. En palabras de la titular del área, "Hicimos esta web... [p]ara hacer de ella la vidriera comercial que aún no teníamos"13 y que, en efecto, la crisis del COVID-19 y las restricciones asociadas, aceleraron como necesidad del sector.

El sitio busca “(...) promover la oferta exportable de Pergamino, estadísticas locales actualizadas y toda la información de los mercados; entre otras cuestiones que son de sumo interés para quienes estén planificando llegar con sus productos a otros ámbitos fuera del país"14. Además, se dispuso en la misma toda la actividad desarrollada durante la pandemia en materia de capacitaciones.

Con posterioridad, a principios del 2021, se realizó una amplia convocatoria para que las empresas de la ciudad se sumen a la oferta exportable de Pergamino que está disponible en dicho sitio. La idea de la Dirección es que las empresas puedan presentar y ofrecer un producto exportable, por ende, la convocatoria no solo estuvo direccionada a quienes estaban exportando en aquel momento sino también, a quienes desean ingresar como oferta disponible que se promociona a través de la Web municipal, recibiendo asesoramiento ad hoc ${ }^{15}$. Así, en reemplazo de estrategias más

\footnotetext{
${ }^{12}$ Pergamino será partícipe del Foro Mundial de Ciudades y Plataformas Logísticas (3 diciembre 2020). Periódico La OPINIonline. Recuperado de: https:/laopinionpergamino.com.ar/nota/46566/2020/12/pergamino-sera-participe-del-foro-mundial-de-ciudades-y-plataformas-logisticas.

${ }^{13}$ Comercio Exterior lanzó su página web oficial (18 noviembre 2020). Diario El Tiempo. Recuperado de: http://www.eltiempodepergamino.com.ar/12226-generales-comercio-exterior-lanzo-su-pagina-weboficial

${ }^{14}$ Convocan a que las empresas pergaminenses se sumen a los nuevos programas de exportación (30 enero 2021). Periódico La OPINIonline. Recuperado de: https://laopinionpergamino.com.ar/nota/48534/2021/01/convocan-a-que-las-empresas-pergaminenses-se-sumen-a-los-nuevos-programas-deexportacion

15 Ídem
} 
convencionales de promoción del comercio exterior, imposibles de materializar por la pandemia, fueron readaptadas a las nuevas modalidades de comunicación.

De esta manera, la pandemia habilitó en Pergamino un fortalecimiento de las estrategias unilaterales para la promoción del comercio exterior local, donde la extendida digitalización de procesos e intercambios, fue la variable global más aprovechada por el distrito en su actividad de promoción, pero también en la posibilidad de participar en espacios y encuentros que le permitan nutrirse de conocimiento en el mismo campo temático. Sin lugar a dudas, su trayectoria de elevada internacionalización es un componente central de su performance en los meses bajo estudio, donde toda esa expertise se puso en práctica para la búsqueda de oportunidades y las readaptaciones iniciadas ante un contexto adverso.

\section{Tandil}

Tandil es un municipio de tamaño intermedio ubicado en el centro-sureste de la Provincia de Buenos Aires, limitando con los partidos de Rauch, Azul, Ayacucho, Balcarce, Lobería, Necochea y Benito Juárez. Se sitúa en la pampa-húmeda, sobre el "Sistema de Tandilia", un conjunto de sierras que se extienden desde el partido de Olavarría hasta la ciudad de Mar del Plata y se encuentra a aproximadamente $360 \mathrm{~km}$ de la CABA. Cuenta con una población de 123.871 habitantes, radicados en su mayoría en la ciudad cabecera homónima, y luego en las restantes localidades y su zona rural (INDEC, 2010).

Su estructura económica y productiva es diversificada, destacándose en este aspecto la actividad agrícola, ganadera, el turismo (gastronomía, hotelería, comercios), la industria metalmecánica y la alimenticia ${ }^{16}$. A su vez, dispone de un Parque Científico Tecnológico (PCT), creado en el año 2003, cuyo objetivo es generar las condiciones propicias para crear, radicar y/o fortalecer empresas innovadoras de base tecnológica y facilitar los procesos de transferencia de conocimientos y tecnologías al medio productivo. El PCT depende de la Universidad Nacional del Centro de la Provincia de Buenos Aires (UNICEN) y se nuclea en la Cámara de Empresas del Polo Informático de Tandil (CEPIT), la cual está constituida aproximadamente por cincuenta empresas, en su mayoría locales (Mutti, 2018).

De igual manera, debido a su estratégica localización, Tandil se ve atravesada por la Ruta Nacional 226 (vincula a Tandil con Mar del Plata y el noroeste de la Provincia), la Ruta Provincial 30 (conecta a Tandil con la CABA) y la Ruta Provincial 74 (enlaza a la ciudad con el este provincial). Así, el municipio dispone de importantes lazos comerciales con diferentes centros productivos a través de puertos marítimos de la provincia tales como Dock Sud y Buenos Aires $(430 \mathrm{~km})$, La Plata $(390 \mathrm{~km})$, Bahía Blanca (370 km), Mar del Plata (170 km) y Quequén (165 km) (Blas, 2017).

De acuerdo a lo establecido por Calvento y Carcedo (2016) “(...) la política internacional subnacional desplegada por el Municipio de Tandil ha permitido catalogarlo desde sus inicios como un gobierno municipal de participación internacional

\footnotetext{
${ }^{16}$ www.tandil.gov.ar
} 
muy alta” (p.78), evidenciando el despliegue continuado de estrategias unilaterales, bilaterales y multilaterales.

En el año 2006 se constituyó la primera vinculación formal internacional de la historia tandilense, rubricándose un convenio de hermanamiento con la Intendencia Municipal de Montevideo (Uruguay) (Blas, 2017), tras la firma de la "Declaración Marco Sobre Cooperación", convalidada por Ordenanza N ${ }^{\circ} 10421 / 2006$. Desde entonces, se multiplicaron las estrategias de este tipo, particularmente con contrapartes italianas, logrando un total de cinco hermanamientos (Mutti, 2018).

En lo que respecta a las relaciones multilaterales, en 2004 se materializó la incorporación de Tandil como miembro pleno de la Red de Mercociudades. Su accionar como sede de encuentros de la Red, se mantuvo desde 2005, así como para el desarrollo de una reunión de la Sección Nacional del Foro Consultivo de Municipios Estados Federados, Provincias y Departamentos del MERCOSUR y del Consejo de Mercociudades, lo que reforzó el posicionamiento de la ciudad en ambas redes. En efecto, la participación activa del municipio en Mercociudades, se vincula también a su rol en el FCCR (Calvento y Carcedo, 2016). También cuenta en su haber con un importante involucramiento en otras redes globales y regionales como fue el Programa URB-AL (hasta 2013), en la Unión de Ciudades Capitales Iberoamericanas (UCCI), CGLU, la Red InterLocal (Mutti, 2018) y la UIM.

En relación a las estrategias unilaterales, se establece como característica general que las políticas locales de comercio exterior se han englobado en un programa integral de promoción de exportaciones e importaciones, abarcando un amplio conjunto de estrategias que pueden ser agrupadas en tres tipos de herramientas de asesoramiento: a) capacitación e información a las empresas locales, de manera individual o en acuerdo con agencias provinciales y nacionales (Cancillería, Secretaría de la Pequeña y Mediana Empresa y los Emprendedores -SEPYME-, entre otras); b) régimen propio de promoción de las exportaciones -que otorga asistencia para participación en ferias, rondas de negocios, exposiciones, misiones comerciales- reglamentado bajo las Ordenanzas $\mathrm{N}^{\mathrm{o}} 5166$ y $\mathrm{N}^{\mathrm{o}} 7716$; y c) apoyo institucional directo o indirecto de la Fundación ExportAr, contando con una oficina local (Oficina de Vinculación Internacional) que comparte espacio con el gobierno municipal (Calvento y Carcedo, 2016).

Sumado a aquellas actividades relacionadas al comercio exterior, el Municipio de Tandil se ha destacado por la presencia de visitas protocolares de representantes diplomáticos de diferentes países con el foco puesto, principalmente, en el intercambio económico, las posibilidades comerciales y la cooperación internacional. Entre ellas se pueden mencionar, la visita del embajador de la India (2014); del embajador de Estados Unidos, Chile y Montenegro (2015), del Cónsul de Bolivia también ese año (Calvento y Carcedo, 2016); del representante comercial de Taiwán en Argentina (2016); de funcionarios de la comuna de Santeramo in Colle (2017) y de la Reina Margarita II y Príncipe Federico de Dinamarca y el embajador de este país (2019). 


\section{La internacionalización de Tandil en tiempos pandémicos}

La actual gestión municipal de Tandil, a cargo del Dr. Miguel A. Lunghi, perteneciente a la coalición Juntos por el Cambio, gobierna el distrito de manera ininterrumpida desde el año 2003, aspecto ineludible para comprender algunas líneas de continuidad de sus políticas locales en todos los ámbitos, incluido el de las relaciones internacionales.

A partir de 2004, con la creación de la Oficina de Vinculación Internacional en su organigrama, se produjo un punto de inflexión en materia de impulso institucional a la política internacional subnacional, la cual se fue jerarquizando con posterioridad. En el período que nos ocupa, el Municipio cuenta con una Secretaría de Desarrollo Productivo y Relaciones Internacionales, cuyas funciones están dirigidas a “(...) promover, orientar, asesorar y apoyar a los distintos sectores económicos de la comunidad. Para ello, la Secretaría está organizada en siete programas de trabajo transversales a los sectores económicos, los cuales son observatorio territorial, creación de empresas, innovación, financiamiento, fomento de negocios, inserción laboral y relaciones internacionales" ${ }^{\prime 17}$. En este sentido, la secretaría cuenta a su vez, con la Dirección de Industria, Comercio y Fomento de Negocios, la Dirección de Asuntos Agropecuarios y finalmente, la Dirección de Turismo. El perfil formativo de la titular de la Secretaría también representa un indicador de la profesionalización del área ${ }^{18}$.

Como se observará a continuación, en pleno contexto de pandemia, y a diferencia del caso de Pergamino, el grueso de las actividades que conforman la agenda municipal en materia de internacionalización, se ha inclinado, principalmente, hacia las relaciones multilaterales y la participación en redes de ciudades.

Por un lado y debido al activismo desplegado por Tandil en Mercociudades, en diciembre del año 2020, al cumplirse los 25 años de su fundación, Tandil asumió la presidencia de la institución durante el período 2020-2021, convirtiéndose en la primera ciudad intermedia en presidir la organización de gobiernos locales más importante del continente. Debido a la pandemia, la $25^{\circ}$ Cumbre Anual de la Red fue organizada por Tandil, en modalidad virtual, a través de la Cámara de Empresas del Polo Informático de Tandil (CEPIT), interpretándose como "(...) una gran oportunidad para mostrarle al resto de las ciudades la importancia de nuestro sector informático y del conocimiento" en palabras de su intendente municipal ${ }^{19}$. En esa ocasión, también se definieron nuevas autoridades, se incorporaron nuevas ciudades a la Red y se consensuaron cuatro líneas concretas de trabajo en el contexto de pandemia, materializadas en la "Declaración de Tandil" emergente de la Cumbre: profundizar la autonomía local; avanzar en la gestión asociada; mejorar el acceso a financiamiento de los gobiernos locales; y continuar trabajando con vocación integradora. De hecho, el Plan de Trabajo de la Presidencia de Tandil 2020-2021, reconoce como objetivo central reafirmar y valorizar la gestión asociada entre los gobiernos y la sociedad civil.

\footnotetext{
17 www.tandil.gov.ar

${ }_{18}$ La actual secretaria de Desarrollo Productivo y Relaciones Internacionales es Historiadora, tiene un Máster en Ciencias Sociales de la Facultad Latinoamericana de Ciencias Sociales (FLACSO). Además, es especialista en gestión del Comercio Exterior en el Instituto Valenciano de la Exportación.

${ }^{19}$ Cumbre de Mercociudades se realizará virtualmente con sistema desarrollado en Tandil (24 noviembre 2020).Diario La Voz de Tandil. Recuperado de: https://www.lavozdetandil.com.ar/2020/11/24/cumbrede-mercociudades-se-realizara-virtualmente-con-sistema-desarrollado-en-tandil
} 
En el marco de este liderazgo institucional regional, Tandil asumió responsabilidades múltiples como por ejemplo, la organización de una ronda comercial multisectorial para facilitar un primer contacto entre los representantes de las empresas de las más de 360 ciudades de la región sudamericana que conforman la red ${ }^{20}$; la organización de la Cumbre de Mercociudades que en abril de 2021 permitió la firma de acuerdos entre la red y diversos gobiernos locales de la región, que accedieron a financiamiento para la implementación de proyectos de cooperación sur-sur seleccionados en base a una convocatoria ad hoc; así como presidir el evento internacional sobre Cambio Climático e Innovación, Innovate4Cities Conference (I4C 2021), que fue desarrollado virtualmente.

Otro espacio multilateral en el que Tandil se involucró desde su nacimiento, fue en Cities for Global Health, reseñado en segmentos previos. En el siguiente gráfico se puede evidenciar el nivel de participación de los municipios argentinos en esta plataforma, medido por la cantidad de iniciativas compartidas, encabezando en el global, CABA con 24 iniciativas, y en segundo lugar, dentro de la jurisdicción bonaerense, el municipio de Tandil con 7 líneas de gestión relacionadas a respuestas a la pandemia en materia de asistencia alimentaria, protocolos de aislamiento, comités de seguimiento de la crisis sanitaria, entre otros.

\section{Gráfico 1: Ranking de participación de gobiernos locales argentinos en Cities for}

\section{Global Health medido por cantidad de iniciativas compartidas}

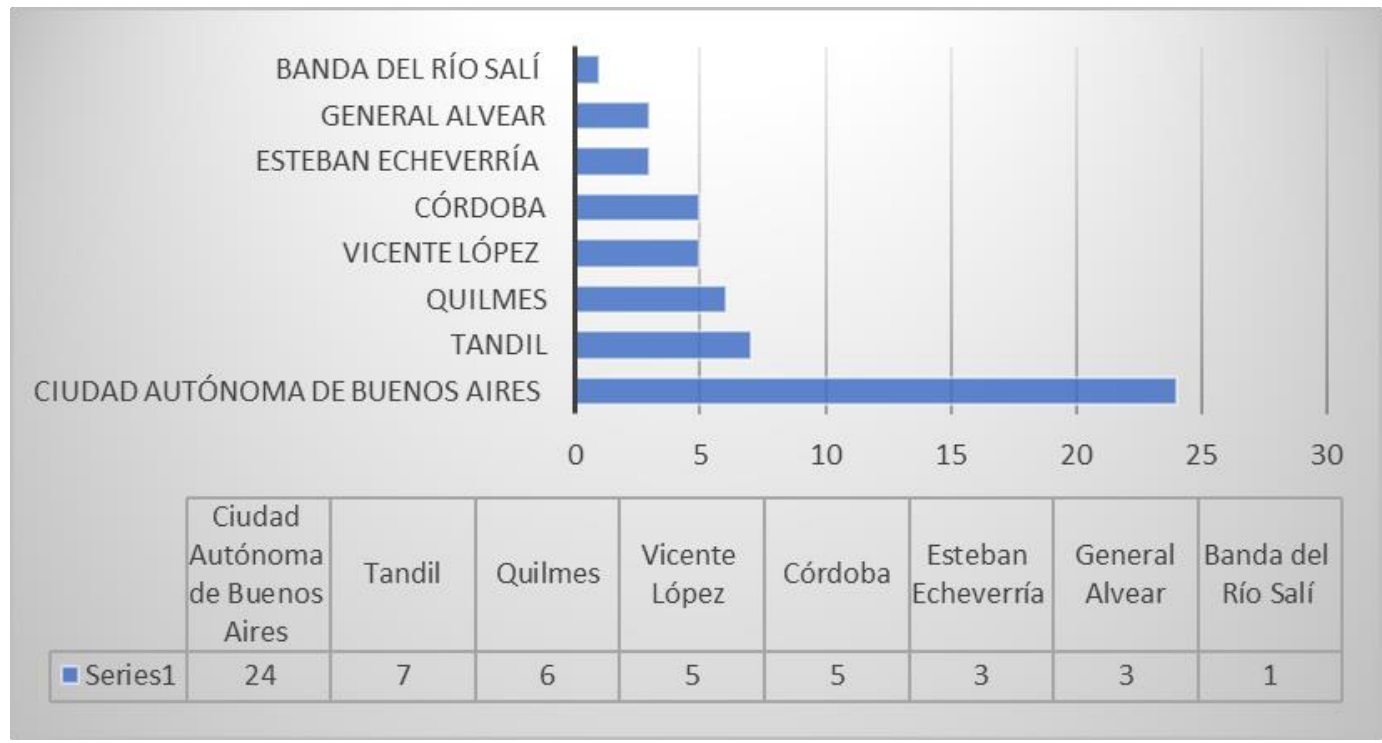

Fuente: elaboración propia en base a datos extraídos de https://www.citiesforglobalhealth.org/

\footnotetext{
${ }^{20}$ Mercociudades: Tandil organiza ronda comercial (4 septiembre 2021). Diario Nueva Era. Disponible en: https://www.nuevaera.ar/nota-mercociudades-tandil-organiza-ronda-comercial-36365
} 
En otro orden de cosas, se ha visualizado una participación de la ciudad en diálogos, intercambios y espacios formativos internacionales atinentes a temáticas como migraciones, refugio y protección internacional, juventudes, resiliencia y sostenibilidad, entre otros. Algunas de ellas organizadas por organismos internacionales, consorcio de actores internacionales o redes de ciudades.

Tabla $N^{\circ}$ 2: Participación del Municipio de Tandil en foros y espacios internacionales multilaterales durante el año 2020

\begin{tabular}{|l|c|}
\hline \multicolumn{1}{|c|}{ Tema } & Año \\
\hline Participación del Foro Urbano Mundial en los Emiratos Árabes Unidos. & 2020 \\
\hline $\begin{array}{l}\text { Intervención del Intendente Lunghi en la consulta a Gobiernos Locales de } \\
\text { la Organización de las Naciones Unidas (ONU), en su 75ªniversario. }\end{array}$ & 2020 \\
\hline $\begin{array}{l}\text { Intervención y participación del Intendente Lunghi del Bureau Ejecutivo } \\
\text { de CGLU (Ciudades y Gobiernos Locales Unidos). }\end{array}$ & 2020 \\
\hline $\begin{array}{l}\text { Participación del Conversatorio Virtual sobre Gobernanza Migratoria y } \\
\text { Pandemia de COVID-19 organizado por la Organización Internacional } \\
\text { para las Migraciones (OIM) para América del Sur y el Instituto de } \\
\text { Políticas Públicas en Derechos Humanos del MERCOSUR (IPPDH). }\end{array}$ & 2020 \\
\hline $\begin{array}{l}\text { Participación en el Diálogo Internacional por la Juventud de América: } \\
\text { Primer Encuentro, organizado por la Unidad Temática de Juventudes de } \\
\text { Mercociudades. }\end{array}$ & 2020 \\
\hline $\begin{array}{l}\text { Participación del Encuentro Nacional de Ciudades Solidarias del Alto } \\
\text { Comisionado de las Naciones Unidas para los Refugiados (ACNUR) junto } \\
\text { a OIM. }\end{array}$ & 2020 \\
\hline $\begin{array}{l}\text { Participación e intervención en el "Ciclo de pensamiento global, acción } \\
\text { local", eje "Turismo post pandemia, medidas de recuperación y } \\
\text { resiliencia”, organizado por el Comité de internacionalización de ciudades } \\
\text { del Consejo Argentino para las Relaciones Internacionales (CARI). }\end{array}$ & 2020 \\
\hline $\begin{array}{l}\text { Participación de las jornadas sobre Sostenibilidad Urbana y la Post } \\
\text { Pandemia "Nuevos desafíos y oportunidades para las ciudades", } \\
\text { organizado por Mercociudades con el apoyo de ONU HÁBITAT, CEPAL, } \\
\text { FLACSO, Resilient Cities Network, Fundación AVINA y Asociación } \\
\text { IVY. }\end{array}$ & 2020 \\
\hline $\begin{array}{l}\text { Participación e intervención en el "Foro para la Estrategia latinoamericana } \\
\text { hacia la acción climática", una sesión política de alto nivel para la } \\
\text { organizado por Mercociudades, CGLU, UCCI, ICLEI, FLACMA, AL-Las } \\
\text { y CGMCE }\end{array}$ & 2020 \\
\hline
\end{tabular}

Fuente: elaboración propia en base a sitios varios: sitios oficiales de redes tales como Mercociudades, CGLU, ACNUR y diario ABCHoy. 
En lo que se refiere a las estrategias unilaterales, durante el año 2020, uno de los hechos más destacados que distinguió a Tandil fue la puesta en marcha de la Agencia de Cooperación e Inversiones, una entidad público-privada impulsada de manera conjunta, por el municipio y la UNICEN, en el marco del Acuerdo del Bicentenario ${ }^{21}$, que busca contribuir a la expansión e internacionalización de la economía regional y local y fortalecer las inversiones productivas en el territorio, promoviendo el espíritu empresarial, la innovación y la competitividad ${ }^{22}$. Desde el Acuerdo del Bicentenario también se le dio impulso al proyecto Marca Tandil, una estrategia de marketing territorial de proyección nacional e internacional de la ciudad que, en la actualidad, se encuentra en etapas preliminares de desarrollo ${ }^{23}$.

En el transcurso de 2021, a su vez, con el retorno parcial de algunas actividades presenciales, el municipio participó de la ronda de negocios de empresarios e instituciones tandilenses con los Embajadores de Kuwait y Egipto; recibiendo a estos representantes y articulando reuniones con una veintena de empresas e instituciones tandilenses que plantearon la posibilidad de hacer negocios y establecer acuerdos de financiación para futuros proyectos con dichos países ${ }^{24}$. También realizó reuniones virtuales con representantes extranjeros de distintos países como, por ejemplo, con el Embajador de China en Argentina ${ }^{25}$.

De esta manera, Tandil, contando con un recorrido y trayectorias destacadas de internacionalización en su haber, evidenció un activismo diversificado durante la pandemia, período en el cual, si bien sus relaciones multilaterales y la presidencia de Mercociudades, resultaron lo más significativo en la jerarquización de su agenda de relaciones internacionales, también pudo mostrar un nivel de actividad no desdeñable en materia de acción unilateral.

\footnotetext{
${ }^{21}$ El Acuerdo del Bicentenario (ACB) fue suscripto en el año 2018 a partir de una alianza estratégica entre el Municipio, la Universidad Nacional del Centro de la Provincia de Buenos Aires, las fuerzas políticas con presencia legislativa en el Concejo Deliberante y un amplio conjunto de instituciones que representan a los distintos sectores de la ciudad. A partir de este acuerdo, se impulsó la conformación de un espacio plural, participativo y diverso de pensamiento, planificación y acción, destinado a diseñar las políticas públicas que se concretarán en los próximos años, rumbo a la celebración del 200 aniversario de la ciudad de Tandil en 2023.

${ }^{22}$ Se pone en marcha la Agencia de Cooperación e Inversiones en Tandil (18 de noviembre de 2020).Periódico El Marplatense. Recuperado de: https://elmarplatense.com/2020/11/18/se-pone-enmarcha-la-agencia-de-cooperacion-e-inversiones-en-tandil/

${ }^{23}$ Avanzan con el proyecto para generar la "Marca Tandil" (12 de marzo de 2021). La Voz de Tandil. Recuperado de: https://www.lavozdetandil.com.ar/2021/03/12/avanzan-con-el-proyecto-para-generar-la-marca-tandil-; Se lanza el primer relevamiento de "Marca Tandil" (23 de septiembre 2021). Diario Nueva Era. Disponible en: https://www.nuevaera.ar/nota-se-lanza-el-primer-relevamiento-de-marca-tandil$\underline{36643}$

${ }^{24}$ Culminó la visita de los embajadores de Egipto y Kuwait (18 septiembre 2021). Eldiariodetandil. Recuperado de: https://eldiariodetandil.com/2021/09/18/culmino-la-visita-de-los-embajadores-de-egiptoy-kuwait

${ }^{25}$ El intendente Lunghi se reunió con el embajador de China en Argentina (1 de julio de 2021). Diario El Eco. Recuperado de: https://www.eleco.com.ar/la-ciudad/el-intendente-lunghi-se-reunio-con-elembajador-de-china-en-argentina
} 


\section{Conclusiones}

En este trabajo, de naturaleza exploratoria, se ha presentado un primer acercamiento a la evolución de la agenda de las relaciones internacionales de los gobiernos locales del interior bonaerense en tiempos pandémicos. El estudio se enfrentó con el reto que significa la ausencia de investigaciones científicas generadas en dicha coyuntura sobre la internacionalización de los municipios argentinos, aspecto que reclama un aporte de la academia y del campo disciplinar específico, en función de la gravitación creciente de estos actores en la agenda de las relaciones internacionales contemporáneas.

El análisis de las experiencias bonaerenses de Pergamino y Tandil habilitan algunas conclusiones: a) ambos municipios se sirvieron de su trayectoria de internacionalización, logrando no sólo sostener una agenda activa en este plano, sino también, incluyendo las relaciones internacionales como parte de la actuación local para enfrentar la crisis; b) la coyuntura exigió readaptaciones y esfuerzos de creatividad en la gobernanza local siendo el aprovechamiento de la digitalización y virtualizaciones de muchas de las dimensiones de la gestión pública el elemento más destacable; c) en algunos casos, este último aspecto potenció y elevó los niveles de participación en encuentros bilaterales o espacios multilaterales otrora limitados por costos elevados de desplazamientos físicos; d) dentro de la tipología de estrategias en la que se materializa la política internacional subnacional, mientras Pergamino se recostó más en sus políticas unilaterales de comercio exterior, Tandil mantuvo una performance más integral y diversificada, con énfasis en sus relaciones multilaterales, a través de la participación (y liderazgo institucional) en redes de ciudades.

Ahora bien, la investigación también nos enfrenta a la oportunidad de abrir nuevos interrogantes en la temática. Por un lado, la necesidad de avanzar en la comparabilidad de la performance internacional entre actores municipales con trayectorias previas disímiles, conduce a preguntarse si significó el contexto de pandemia una oportunidad real para aquellos municipios sin previa experiencia en este campo de acción. Del mismo modo, con la intención de trascender la caracterización sobre las modalidades y herramientas empleadas en sus relaciones internacionales, la pregunta emergente es ¿De qué manera estas estrategias impactaron en la gestión local de respuesta o prevención durante la crisis sanitaria? ¿O cómo sirvieron a las políticas transversales de la gestión pública local? Estos, entre otros, constituyen tópicos de la agenda de investigación futura.

\section{Bibliografía}

Alianza Euro-Latinoamericana de Cooperación entre Ciudades y Observatorio de Cooperación Descentralizada (AL-LAS y OCD) (2020). Relatoría del $2^{\circ}$ Webinar de Cooperación Descentralizada y Covid-19. El papel de la acción internacional de los gobiernos locales y la cooperación descentralizada ante la crisis activada por el covid19. Recuperado de: https://proyectoallas.net/2020/05/05/el-papel-de-la-accioninternacional-de-los-gobiernos-locales-y-la-cooperacion-descentralizada-ante-la-crisisactivada-por-el-covid19/ 
Álvarez, M.; Luna Pont, M. y Oddone, N. (2019). América Latina global: estudios regionales sobre paradiplomacia. Tres de Febrero, Argentina: EDUNTREF.

Batista, S., Jakobsen, K. y Evangelista, A. (2008). La apertura al exterior de las ciudades latinoamericanas y la cooperación descentralizada. Montevideo, Uruguay: Observatorio de Cooperación Descentralizada Unión Europea-América Latina.

Blas, M. M. (2017). Estrategias públicas turísticas a escala municipal. El caso de Tandil: 2002-2012. (Tesis de Licenciatura en Turismo, Facultad de Ciencias Humanas, UNICEN). Tandil, Argentina.

Borja, J. y Castells, M. (1997). Local y global. La gestión de las ciudades en la era de la información. Barcelona: UNCHS, Santillana S. S. Taurus.

Calvento, M. (2020) (Comp.). Relaciones Internacionales y Actores Locales: Dinámicas, Estrategias y Políticas en el Interior de la provincia de Buenos Aires. Tandil, Argentna: CEIPIL-UNICEN.

Calvento, M. (2016a) (Comp.). Gestión y política internacional subnacional. El caso de los municipios del interior de la Provincia de Buenos Aires. Tandil, Argentina: CEIPILANPCyT.

Calvento, M. (2016b). La Política Internacional Subnacional: una propuesta para el abordaje del accionar contemporáneo en Argentina. Revista Desafíos, 28 (1), pp. 295332. Doi: dx.doi.org/10.12804/desafios28.1.2016.07

Calvento, M. y Carcedo, J. F. (2016). Capítulo III: El Índice de Participación Internacional y la trayectoria internacional de los municipios intermedios. En Calvento, M. (2016a) (Comp.): Gestión y política internacional subnacional. El caso de los municipios del interior de la Provincia de Buenos Aires. Tandil, Argentina: CEIPILANPCyT. Páginas 71-97.

Calvento, M. (2012). La participación internacional de los estados subnacionales. El caso de los gobiernos municipales de tamaño intermedio de la Provincia de Buenos Aires. Tesis de Doctorado en Ciencia Política. Escuela de Política y Gobierno. UNSAM. CABA, Argentina.

Carignani Sardoy, C. (2017). Política Internacional Subnacional en Argentina (20002015). Tesis de Grado. Escuela de Relaciones Internacionales. Facultad de Ciencias Sociales. Universidad del Salvador. CABA. Argentina.

Castro, L. y Saslavsky, D. (2009). Cazadores de Mercados. Comercio y promoción de exportaciones en las provincias argentinas. Buenos Aires: CIPPEC.

Cities for Global Health. Sitio Oficial: https://www.citiesforglobalhealth.org/

Colacrai, M. (2013). El rol de las ciudades en el actual escenario internacional y en los procesos de integración regional: ni tanto, ni tan poco.... Revista de Integración y Cooperación internacional, 14, 4-20. Recuperado de: http://hdl.handle.net/11336/15308 
Consejo Federal de Inversiones [CFI] (2017). Programa Municipios Internacionales de la Provincia de Buenos Aires. Recuperado de: http://biblioteca.cfi.org.ar/wpcontent/uploads/sites/2/2017/01/pmi-informe-final.pdf

Cravacuore, D. (2015). Las redes de ciudades en Argentina. Revista TEACS, 7, (16), pp. 11-31.

Duchacek, I. (1986). The Territorial Dimension of Politics: Within, Among and Across Nations. London: Westview Press.

Eldiariodetandil (2021). Culminó la visita de los embajadores de Egipto y Kuwait. 18 de noviembre. Recuperado de: https://eldiariodetandil.com/2021/09/18/culmino-la-visitade-los-embajadores-de-egipto-y-kuwait

El Eco (2021). El intendente Lunghi se reunió con el embajador de China en Argentina. 1 de julio. Recuperado de: https://www.eleco.com.ar/la-ciudad/el-intendente-lunghi-sereunio-con-el-embajador-de-china-en-argentina

El Marplatense (2020) Se pone en marcha la Agencia de Cooperación e Inversiones en Tandil. 18 de noviembre. Recuperado de: https://elmarplatense.com/2020/11/18/sepone-en-marcha-la-agencia-de-cooperacion-e-inversiones-en-tandil/

El Tiempo (2020). Comercio Exterior lanzó su página web oficial. 18 de noviembre. Recuperado de: http://www.eltiempodepergamino.com.ar/12226-generales-comercioexterior-lanzo-su-pagina-web-oficial

Hidalgo, E. (2021). La cooperación subnacional entre Argentina y China. Página 12, 12/4/2021. Recuperado de: https://www.clarin.com/opinion/diplomacia-nuevos-marcoscooperacion-internacional_0_ELY87ctY.html

INDEC (2010): Censo Nacional de Población, Hogares y Viviendas 2010. Instituto Nacional de Estadística y Censos. Buenos Aires [en línea]. Recuperado de: https://www.indec.gob.ar/indec/web/Nivel4-CensoNacional-3-7-Censo-2010

Juste, S. (2020). COVID-19: Paradiplomacia y gestión de crisis. Equilibrium Global, 10/6/2020. Recuperado de: https://equilibriumglobal.com/covid-19-paradiplomacia-ygestion-de-crisis/

La OPINIonline (2021). Convocan a que las empresas pergaminenses se sumen a los nuevos programas de exportación. 30 de enero. Recuperado de: https://laopinionpergamino.com.ar/nota/-48534/2021/01/convocan-a-que-las-empresaspergaminenses-se-sumen-a-los-nuevos-programas-de-exportacion

La OPINIonline (2020). Pergamino será partícipe del Foro Mundial de Ciudades y Plataformas Logísticas. 3 de diciembre. Recuperado de: https://laopinionpergamino.com.ar/nota/-46566/2020/12/pergamino-sera-participe-delforo-mundial-de-ciudades-y-plataformas-logisticas.

La Voz de Tandil (2021). Avanzan con el proyecto para generar la "Marca Tandil". 12 de marzo. Recuperado de: https://www.lavozdetandil.com.ar/2021/03/12/avanzan-conel-proyecto-para-generar-la--marca-tandil- 
La Voz de Tandil (2020). Cumbre de Mercociudades se realizará virtualmente con sistema desarrollado en Tandil. 24 de noviembre. Recuperado de: https://www.lavozdetandil.com.ar/2020/11/24/cumbre-de-mercociudades-se-realizaravirtualmente-con-sistema-desarrollado-en-tandil

Maira, L. (2010) (Ed.): La Política Internacional Subnacional en América Latina. Buenos Aires: Libros del Zorzal.

Manzano, F. y Velázquez, G. (2015). La evolución de las ciudades intermedias en la Argentina. En Revista GEO UERJ. Año: 2015. Pp. 258 - 282. Río de Janeiro.

Marcovitch, J. e Dallari, P. (2014). Relações Internacionais de Âmbito Subnacional: A Experiência de Estados e Municípios no Brasil. São Paulo: Instituto de Relações Internacionais-Universidade de São Paulo.

Mercociudades. Mercociudades responde al Covid-19. Sitio oficial: https://mercociudades.org/covid-19/

Michelmann, H. \& Soldatos, P., (1990). Federalism and International Relations. The Role of Subnational Units. Oxford: Clarendon Press.

Municipio de Pergamino (2018). Perfil General del Municipio de Pergamino Argentina. Recuperado de: https://www.pergamino.gob.ar/wpcontent/uploads/2019/07/PERFIL-PERGAMINO-PRODUCCION-2018.pdf

Municipio de Pergamino (2011). Plan Estratégico Pergamino: nos encontramos para crecer. Recuperado de: https://www.mininterior.gov.ar/planificacion/pdf/planesloc/BUENOSAIRES/Plan-Estrategico-Pergamino.pdf

Municipio de Pergamino. Sitio Web oficial: https://www.pergamino.gob.ar/

Municipio de Tandil. Sitio Web Oficial: https://www.tandil.gov.ar

Mutti, A. I. (2018). La política internacional de los actores subnacionales. El Municipio de Tandil como estudio de caso (2003 - 2015). Tesis de Licenciatura en Relaciones Internacionales, Facultad de Ciencias Humanas. Tandil, Argentina.

Nueva Era (2021a). Se lanza el primer relevamiento de "Marca Tandil". 23 de noviembre. Recuperado de: https://www.nuevaera.ar/nota-se-lanza-el-primer$\underline{\text { relevamiento-de-marca-tandil-36643 }}$

Nueva Era (2021b). Mercociudades: Tandil organiza ronda comercial. 4 de noviembre Recuperado de: https://www.nuevaera.ar/nota-mercociudades-tandil-organiza-rondacomercial-36365

Oddone, N. (2021). La para-diplomacia y los nuevos marcos de la cooperación internacional. Clarín, 19 de junio. Recuperado de: https://www.clarin.com/opinion/diplomacia-nuevos-marcos-cooperacioninternacional_0_ELY87ctY.html 
Organización de las Naciones Unidas (ONU) (2020). Documento de políticas: La COVID-19 en un mundo urbano. Julio de 2020. Recuperado de: https://www.un.org/sites/un2.un.org/files/covid-19_in_an_urban_world_spanish.pdf

Pontis (2012). Las áreas internacionales de los municipios argentinos. Disponible en: http://pontisweb.blogspot.com.ar/2012/03/les-presentamos-los-resultados-de.html

Prado Figuereido, D. (2018). A atuação internacional dos governos subnacionais: construções conceituais, limites e contribuições para o caso brasileiro. Revista Carta Internacional, 13 (3), pp. 137-168.

Romero, M. (2004). Una aproximación contextual y conceptual a la cooperación descentralizada. Ilustre. Municipalidad de Valparaíso y Diputación de Barcelona. Valparaíso.

Salomón, M. (2007). La acción exterior de los gobiernos subnacionales y el análisis de políticas exteriores. En $1^{\circ}$ Encontro Nacional da ABRI Política Externa. Brasília (DF), de 25 a 27 de julho de 2007.

Secretaría de Relaciones Económicas Internacionales [SREI] (2015). Buenos Aires al Mundo. Ministerio de Producción, Ciencia y Tecnología. Comunicación Institucional.

Schiavon, J. (2020). La evolución de la Paradiplomacia en México (1980-2020). Trabajos de Investigación en Paradiplomacia 4, (1), pp. 22-35. Recuperado de: https://www.museovirtualug.org/post/c\%C3\%B3mo-ha-evolucionado-laparadiplomacia-en-m\%C3\%A9xico-1980-2020

Vigevani, T. (2004). El marco jurídico e institucional para la gestión internacional de los actores subnacionales gubernamentales en Brasil. Revista Integración y Comercio, 21, pp. 27-46.

Zapata Garesché, E. (2007). Manual práctico para internacionalizar la ciudad. Guía para la acción exterior de los gobiernos locales y la cooperación descentralizada Unión Europea-América Latina. Barcelona: Diputación de Barcelona.

Cómo citar

NICOLAO, J., CALVENTO, M. y PIERSANTI, A. (2022). Pandemia y gobiernos locales en el interior de la provincia de Buenos Aires: el reimpulso internacionalizador. Revista Integración y Cooperación Internacional, 34 (ene-jun), $24-48$ 